\title{
Keloids and hypertrophic scars: a review
}

\author{
J. Meenakshi, ${ }^{1}$ V. Jayaraman, ${ }^{2}$ K. M. Ramakrishnan, ${ }^{3}$ M. Babu ${ }^{1}$ \\ ${ }^{1}$ Biomaterials Division, Central Leather Research Institute, TICEL Bio Park, Taramani, Chennai-600113, India, ${ }^{2}$ Division of \\ Plastic Surgery, Kilpauk Medical College and Hospital, Chennai-600010. India, ${ }^{3}$ Plastic Surgery and Burn Intensive Care Unit, K. \\ K. CHILDS Trust Hospital, Chennai-600034, India
}

Address for correspondence: Mary Babu, Biomaterials Division, Central Leather Research Institute,TICEL BioPark, Taramani, Chennai. 600 113. India, E-mail: marybabu@hotmail.com

\section{KEY WORDS}

Keloids, Hypertrophic scars, Collagen morphology

\section{INTRODUCTION}

eloids and Hypertrophic scars (HSc) are dermal fibroproliferative disorders unique to humans that occur following trauma, inflammation, surgery,

burns and sometimes spontaneously. These are characterized by excesses deposition of collagen in the dermis and the subcutaneous tissues. Contrary to the fineline scar characteristics of normal wound repair, the exuberant scarring of keloid and HSc results typically in disfigurement, contractures, pruritis and pain. Keloids occur in individuals with a familial disposition among the Blacks, Hispanics and Orientals, they enlarge and extend beyond the margins of the original wound and rarely regress [Figure 1]. HSc are raised, erythematous, pruritic, fibrous lesions that typically remain within the confines of the original wound, usually undergo at least partial spontaneous resolution over widely varying time courses, and are often associated with contractures of healing tissues [Figure 2].

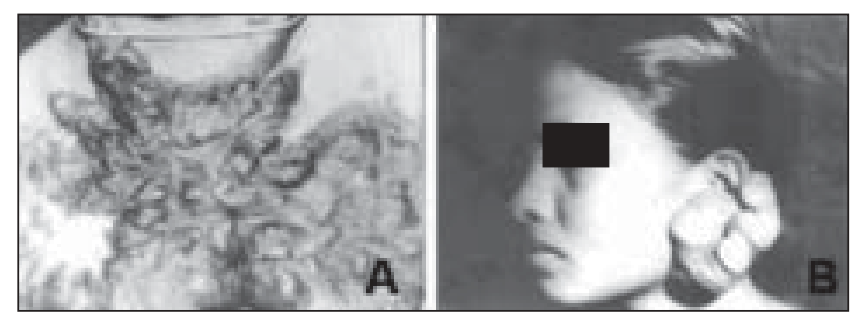

Figure 1: Post burn keloid (A) and ear lobule keloid (B)
These disorders represent aberrations in the fundamental processes of wound healing, which include cell migration and proliferation, inflammation, increased synthesis and secretion of cytokines and extra cellular matrix (ECM) proteins and remodelling of the newly synthesized matrix. Conceptually, it is expected that the wound healing should lead to regeneration of the injured skin; however, adult human healing occurs by the formation of scar, characterized by disordered architecture, which, in the case of keloid and HSc, is also associated with excessive deposition of matrix proteins [Figure 3]. In this review an attempt has been made to summarize the physical, ultra

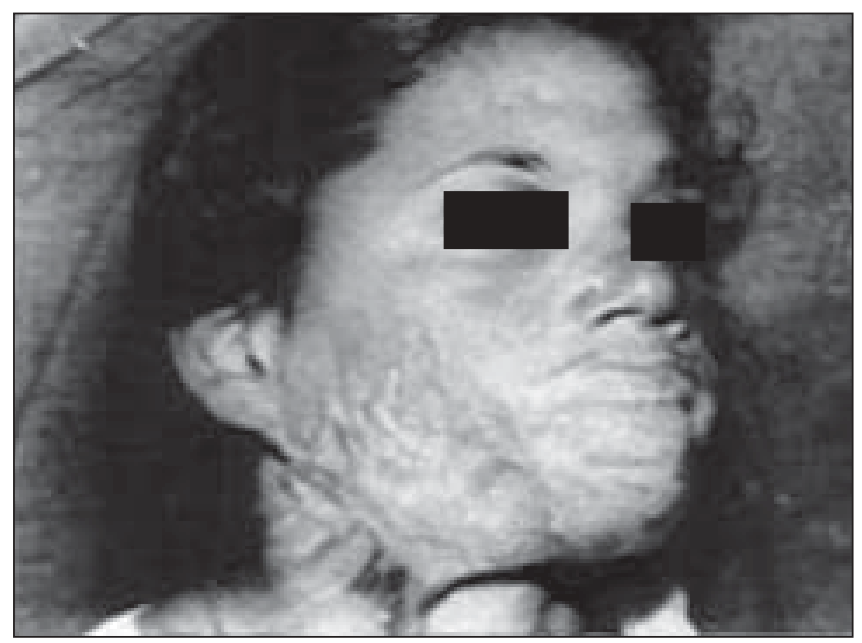

Figure 2: Post burn hypertrophic scar 
structural and molecular aspects of these abnormal scars.

\section{Histological appearance of abnormal scars}

In dermal wound healing, injury to the reticular layer of the dermis is known to contribute to the formation of keloids and HSc. This region mainly consists of collagen and fibroblasts. Histologically the collagen bundles in the dermis of normal skin appear relaxed and are arranged in a random array. Keloids and HSc have collagen bundles that appear stretched and aligned in the same plane as the epidermis. These collagen bundles are thicker and more abundant in keloids and form acellular node-like structures in the deep dermal portion. The centre of the keloid lesion has relatively few cells compared to HSc. Apoptosis has been suggested to be involved in the clearance of some of these cells. In contrast islands composed of aggregates of fibroblasts, small blood vessels and collagen fibres are seen throughout the dermis of HSc. There are significant differences in the epidermal portion of these scars. In case of HSc the epidermis is much thicker than that of normal skin, while keloids show a clear lack of epidermal ridges [Figure 4a \& b]. Immunohistochemical analysis done by various workers has revealed that HSc contains whorls of connective tissue in nodular structures containing $\alpha$ -

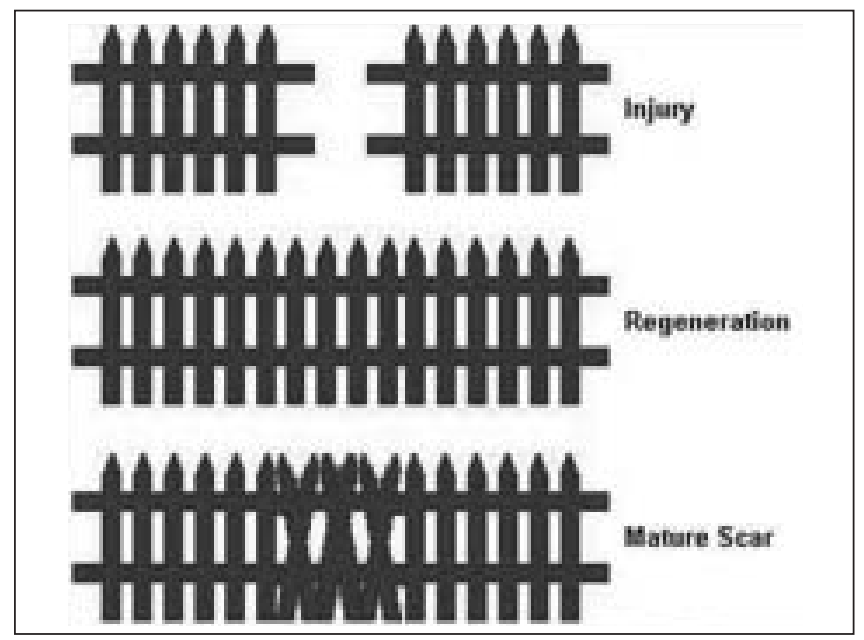

Figure 3: Repair of dermal injury is analogous reconstruction of a picket fence post injury
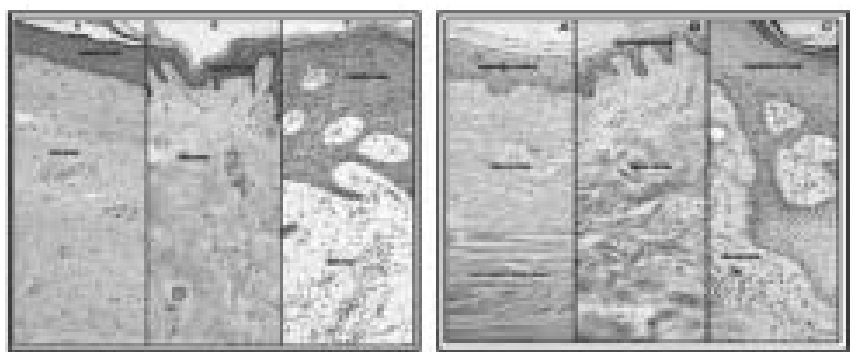

Figure 4: Haematoxylin and eosin staining (A) and vanGeisson's staining (B) of paraffin embedded sections of keloid, normal skin and hypertrophic scar smooth muscle actin positive fibroblasts with small blood vessels and fine, randomly oriented collagen fibrils, whereas keloids have few if any $\alpha$-smooth muscle actin positive fibroblasts and large, thick collagen fibres. ${ }^{1}$ Apart from collagen the other major ECM component is the proteoglycan family. This family consists of large and small proteoglycans which are essential for the fibril formation and alignment of collagen fibrils. Immunohistochemical analysis of various types of Proteoglycans has shown their excess deposition and differential deposition in the abnormal scars. The proteoglycan content and synthesis has been discussed later in this review.

\section{Pathogenesis of abnormal scars}

Normal wound repair involves several well orchestrated phases. Immediately after wounding, platelet degranulation and activation of the complement cascade begins and a fibrin clot for haemostasis is formed which functions as a provisional matrix. platelet degranulation is responsible for the release and activation of an array of potent cytokines, including epidermal growth factor (EGF) ${ }^{2}$, Insulin like growth factor-I (IGF-I) ${ }^{3}$, platelet derived growth factor (PDGF) $)^{4,5}$ and transforming growth factor (TGF- $\left.\beta\right)^{6}$,

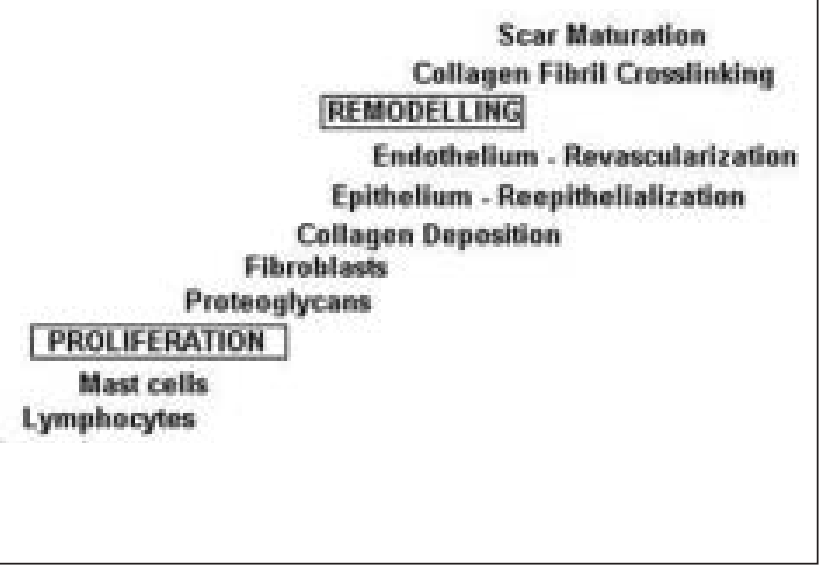

Figure 5: The phases of normal wound repair follow an orderly sequence of events that are regulated by chronological appearance of a number of different cell types over the course of healing. Prolonged activity or abnormal levels of fibrogenic cytokines released during the inflammatory phase may lead to fibroproliferative disorders

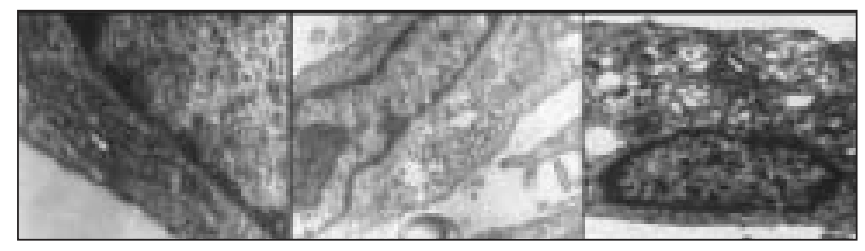

Figure 6: Electron microscopy analysis of dermal fibroblasts isolated from keloid (A), normal skin (B) and hypertrophic scar (C). Arrows indicate endoplasmic reticulum 
which function as chemotactic agents for the recruitment of neutrophils, macrophages, epithelial cells, mast cells, endothelial cells and fibroblasts. This phase of wound healing is called the inflammatory phase. Following this is the proliferative phase which involves the proliferation and differentiation of various inflammatory cells and formation of granulation tissue. All these phases of wound healing have been summarized in [Figure 5]. Prolonged inflammatory stage in large wounds such as a burn or following an infection exaggerates the inflammatory phase of healing leading to increase in the activity of fibrogenic cytokines such as IGF-I and TGF- $\beta$, thereby increasing the risk of development of abnormal scars.

Transformation of a wound clot into granulation tissue requires matrix degradation and biosynthesis that are balanced to achieve optimal wound healing. The degradation of ECM is through the action of collagenase, proteoglycanases and other proteases, which are released by mast cells, macrophages, endothelial cells and fibroblasts. Either excessive synthesis of collagens, fibronectin and proteoglycans by fibroblasts or deficient matrix degradation and remodelling may lead to abnormal wound healing which results in the formation of keloids and $\mathrm{HSc}^{7,8}$

\section{Biochemical analysis of abnormal scars}

Several groups have studied the biochemical and molecular composition of the abnormal scars. Our group has been involved in this type of study for the past several years. We have studied collagen, proteoglycan and water content of the keloids and HSc and compared with normal skin. Total collagen content was measured by hydroxyproline estimation method, ${ }^{9}$ the proteoglycan content was measured by glucosamine estimation method $^{10}$ and the water content was measured as a difference between the wet weight and dry weight of the scar biopsies. Keloid tissue showed high levels of all collagen, proteoglycan and water [Table 1]. The total collagen was fractionated into acid soluble and pepsin soluble portions and the fractionated collagen was again estimated. Interestingly, here keloids show higher acid collagen than the pepsin soluble collagen. The HSc and normal skin show higher pepsin soluble collagen [Table 2]. These observations show that though keloids show high amounts of collagen its cross linking is very poor as the Pepsin soluble fraction represents the cross-linked collagen. Apart from collagen and proteoglycans, the synthesis of other ECM proteins has also been found to be much higher in keloids and HSc.

High rate of production of ECM components indicates highly active fibroblasts. Therefore, the activity of dermal fibroblasts isolated from keloids, HSc and normal skin were studied using ${ }^{3} \mathrm{H}$-thymidine incorporation and estimation of total protein content at various time points. This study showed that both Keloid and HSc fibroblasts are much more active than normal dermal fibroblasts. Between the keloid and HSc fibroblasts, the keloidal ones are more active as shown in Table 3. To confirm the high state of metabolic activity of the keloid and HSc fibroblasts, these fibroblasts were analyzed by electron microscopy. The analysis of detailed cytoplasmic architecture showed the presence of increased endoplasmic reticulum suggesting a high rate of synthesis of the ECM proteins [Figure 6]. ${ }^{11}$

Excess matrix accumulation can occur not only by increased synthesis of ECM components but also by a reduction in matrix degradation, either intracellular or extracellular. The ability of collagenases isolated from the scar fibroblast to degrade collagen has been studied with respect to HSc and it has been shown that the activated HSc fibroblasts have reduced ability to degrade collagen. ${ }^{12}$

\section{Role of cytokines or growth factors in abnormal scar formation}

The release and activation of growth factors during the inflammatory phase of healing are pre-requisites for subsequent processes, including angiogenesis, reepithelialization, recruitment and proliferation of fibroblasts and matrix deposition. Angiogenesis is stimulated by endothelial chemo-attractants and mitogens that are released by mast cells, neutrophils, macrophages and

Table 1: Biochemical Composition of Scars

\begin{tabular}{lccc}
\hline Tissue & Water content & Collagen & Glucosamine \\
Normal skin & $65 \%$ & $50 \%$ & $1.8 \%$ \\
Hypertrophic scar & $70 \%$ & $52.4 \%$ & $2.2 \%$ \\
Keloid & $75 \%$ & $58.2 \%$ & $0.95 \%$ \\
\hline
\end{tabular}

Table 2: Extractability of collagen from normal skin, hypertrophic scar and keloid

\begin{tabular}{lccc}
\hline Tissue & $\begin{array}{c}\text { Neutral } \\
\text { salt sol. }\end{array}$ & $\begin{array}{c}\mathbf{0 . 5} \text { M acetic } \\
\text { acid }\end{array}$ & $\begin{array}{c}\text { Pepsin } \\
\text { treatment }\end{array}$ \\
Normal skin & $5 \%$ & $35 \%$ & $50 \%$ \\
Hypertrophic scar & $7 \%$ & $40.5 \%$ & $43 \%$ \\
Keloid & $8.5 \%$ & $48 \%$ & $40 \%$ \\
\hline
\end{tabular}

Table 3: Estimation of DNA synthesis and total protein content in dermal fibroblasts

\begin{tabular}{lcccccc} 
& \multicolumn{3}{c}{ DNA synthesis } & \multicolumn{3}{c}{ Estimation of total } \\
& $\begin{array}{lcccccc} \\
\text { (3H-thymidine incorporation) }\end{array}$ & \multicolumn{2}{c}{ protein $(\boldsymbol{n g} / \mathbf{m l})$} \\
Tissue & $24 \mathrm{hr}$ & $48 \mathrm{hr}$ & $96 \mathrm{hr}$ & $24 \mathrm{hr}$ & $48 \mathrm{hr}$ & $96 \mathrm{hr}$ \\
Normal skin & 213 & 319.5 & 575.1 & 247 & 395.6 & 712.2 \\
Hypertrophic scar & 285 & 456 & 925.3 & 2.85 & 4.56 & 7.52 \\
Keloid & 3.72 & 5.97 & 9.87 & 4.01 & 6.42 & 10.52 \\
\hline
\end{tabular}

Indian J Plast Surg July-December 2005 Vol 38 Issue 2 
keratinocytes. Wound re-epithelialization occurs following the migration of epithelial cells from the wound margin and epidermal appendages within the wound bed and has been shown to be enhanced by EGF, TGF- $\beta$, vaccinia growth factor and IGF-I. ${ }^{13,14}$ Fibroblast recruitment, proliferation and production of ECM are influenced by the fibrogenic growth factors PDGF, IGF-I and TGF- $\beta$ as well as basic fibroblast growth factor. ${ }^{14,15}$ These fibrogenic growth factors up regulate ECM production, increase the rate of proliferation and/or migration of fibroblast, and inhibit production of proteases required to maintain the balance between production and degradation [Figure 7].

TGF- $\beta$ was initially isolated from human platelets ${ }^{6}$ but has since been shown to be produced at wound site by infiltrating lymphocytes, macrophages and fibroblasts. The TGF- $\beta$ family consists of at least five highly conserved peptides, with TGF- $\beta_{1}$, TGF- $\beta_{2}$, and TGF- $\beta_{3}$ being the principle mammalian forms. Many biological actions of TGF$\beta$ contribute to the normal wound-healing processes and have been implicated in a wide variety of fibrotic disorders. ${ }^{16}$ The release of TGF- $\beta$ by platelets localizes it in the wound environment very soon after injury, where it acts as a chemo-attractant. For neutrophils, T Lymphocytes, monocytes and fibroblasts. The auto induction of TGF- $\beta$ production by fibroblasts in the wound environment may contribute to fibrosis and wound contraction by increasing the production of collagen, fibronectin and proteoglycans ${ }^{17,18}$ and decreasing the production of tissue inhibitors of matrix metalloproteinases (TIMP) I and II and $\alpha 2$ macroglobulin. ${ }^{19}$ In vivo stimulation of granulation tissue formation and enhanced connective tissue response support the role of TGF- $\beta$ in normal wound healing;

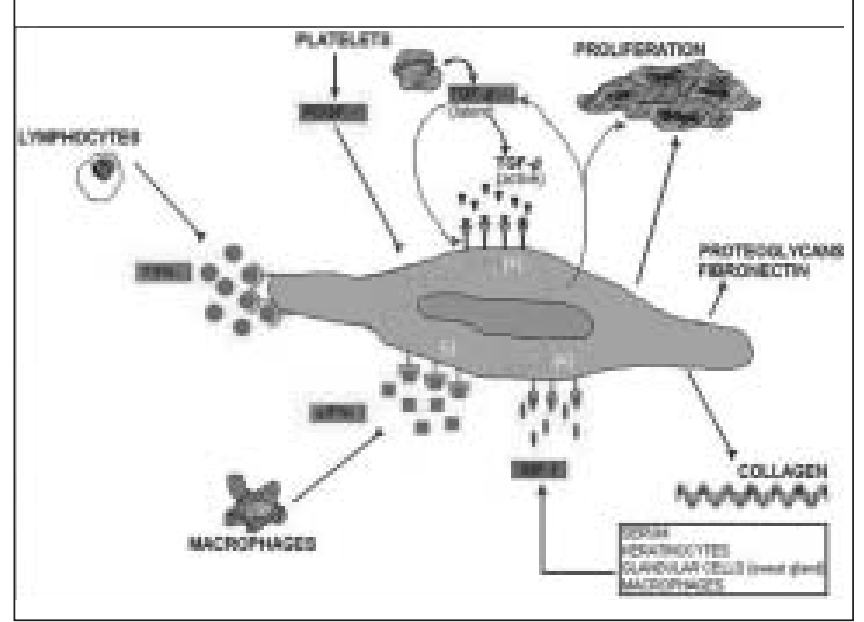

Figure 7: Fibrogenic growth factors including TGF-B, PDGF, and IGF-I are involved in the development of fibroproliferative disorders with interferones being potential inhibitors of matrix synthesis and fibroblast proliferation however, the prolonged and excessive presence of TGF- $\beta$ possibly contributes to the development of keloids and HSc. ${ }^{12,20}$ The inter relationship of the three isoforms of TGF$\beta$, the release and subsequent activation of TGF- $\beta$ from its binding proteins, the synergistic and antagonistic interplay with other growth factors and the extra cellular matrix itself require further investigation. Elevated systemic plasma levels of TGF- $\beta$ have been found to predict the development pulmonary and hepatic fibrosis ${ }^{21}$ and elevated levels of TGF$\beta$ have been found in burn patients with substantial amounts of HSc after injury. ${ }^{22}$ These features suggest a systemic response to injury as well as local factors may be important in the development of dermal fibrosis.

PDGF another important cytokine is also released into the wound by platelets at the early stages of wound healing. In the later stages it is released by infiltrating macrophages, endothelial cells and fibroblasts. PDGF also functions as a chemo-attractant and mitogens for fibroblasts and endothelial cells. ${ }^{5}$ Although abnormal presence of PDGF has not been correlated with the development of abnormal scars, its ability to modulate the production of IGF-I by fibroblast and endothelial cells may contribute to fibrosis. Like TGF- $\beta$ and PDGF, many other growth factors have been implicated in the development of fibrotic disorders. A complete study of all these growth factors will enable us to develop a suitable therapeutic intervention for the treatment of the abnormal scars.

\section{Conventional treatments for abnormal scars based on laboratory findings}

In the past, several drugs have been investigated for the purpose of inhibiting collagen synthesis and accelerating the removal of excessive collagen deposited in the keloids and HSc. Historically; these drugs have included collagen cross-linking inhibitors, $\beta$-aminoproprionitrile (BAPN) and penicillamine, the antimicrotubular agent colchicine and corticosteroids, which interfere with protein synthesis. Treatment of keloids intralesionally with corticosteroid injections, used individually or in combination with surgery, radiation, laser or pressure therapy, and/or silastic gel sheeting, often have an unsatisfactory outcome, frustrating both clinicians and patients. We have found that in case of ear lobule keloids surgery followed by radiation is very effective and in almost all cases treated in this fashion have not shown any recurrence..$^{23}$ The basis of this therapy is decreased rate of cell proliferation following radiation. However other keloids are still quite resistant to any kind of treatment. In spite of recent advancement in therapeutic designs for fibroproliferative disorders, further study is 
required to establish efficacy, timing and optimum dosage of these potential agents for clinical application. In addition most of the target agents are produced by cells during skin wound repair, and their temporal and spatial expression during normal wound healing is required. Therefore, precise intervention will be required for beneficial treatment of pathological scarring.

\section{Future directions for potential therapy}

Difficulty in the treatment of keloids and HSc arises from complexity of cellular and molecular biology of lesions themselves. Increased understanding at this level will lead to the development of new therapies. Control of fibrogenic growth factor effects by monoclonal antibody techniques and receptor antagonists and the development of antisense oligonucleotide therapy offer substantial potential. Appreciation of the immunological response to injury and the regulation of wound healing by the immune system will allow specific growth factor therapy to provide potential down regulatory signals, which some but not all individuals possess after wounding, thereby modifying the whole body response to injury. Finally, with intense pursuit of skin replacements and the enhanced understanding of the role of the dermis in controlling scar contractures and hypertrophy, skin replacement will likely provide new therapies previously unavailable for patients with keloids and HSc.

\section{REFERENCES}

1. Ehrlich HP, Desmoulier A, Diegelmann RF. Morphological and immunochemical differences between keloid and hypertrophic scar. Am J Pathol 1994;145:105.

2. Oka Y, Ort DN. Human plasma epidermal growth factor/burogastrone is associated with blood platelets. J Clin Invest 1983;72:249.

3. Karey KP, Sirkasku DA. Human platelet derived mitogens II subcellular localization of insulin like growth factor- 1 to the $\alpha$ granule and release in response to thrombim. Blood 1989;74:1093.

4. Kohler A, Lipton A. Platelets as a source of fibroblast growth promoting activity. Exp Cell Res 1974;87:297.

5. Ross R, Glonset J A, Kariya B. A platelet dependent serum factor stimulates the proliferation of arterial smooth muscle cell in vitro. Proc. Natl Acad Sci USA 1974;71:1207.
6. Assoian RK, Komoriya A, Meyer CA. Transforming growth factorb inhuman platelets. Identification of a major storage site, purification and charecterisation. J Biol Chem 1983;258:7155.

7. Nedelec B, Tredget EE, Ghahary A. The molecular biology of wound healing following thermal injury: The role of fibrogenic growth factors. In Critical Care of the Burn Patient. Barcelona, SpringerVerlag. 1996.

8. Raghow $\mathrm{R}$. The role of extra cellular matrix in post inflammatory wound healing and fibrosis. FASEB J 1994;8:823.

9. Woessne JF Jr. The determination of hydroxylproline in tissue samples containing small portions of this amino acid. Arch Biochem Biophys 1961;93:440.

10. Elson LA, Morgan WTJ. Colourumetric method for the determination of glucosamine and chondrosamine. Biochem $\mathrm{J}$ 1933;27:1824.

11. Meenakshi J, Jayaraman V, Ramakrishnan KM, Babu M. Ultrastructural differentiation of abnormal scars. Annals of burns and fire disasters 2005;18:83.

12. Ghahary A, Shen YJ, Scott PJ. Enhanced expression of mRNA for transforming growth factor-b, type I and type III procollagen in human post-burn hypertrophic scar tissues. J Lab Clin Med 1998;122:465.

13. Ando $Y$, Jense PJ. Epidermal growth factor and insulin like growth factor I enhance keratinocyte migration. J Invest Dermatol 1993;100:633.

14. Kiristy CP, Lynch SE. Role of growth factors in cutaneous wound healing. A review. Crit Rev Oral Biol Med 1993;4:729.

15. Kovacs EJ, Dipietro LA. Fibrogenic cytokines and connective tissue production. FASEB J 1994;8:854.

16. Border WA, Noble N A. Transforming growth factor $b$ in tissue fibrosis. N. Engl J Med 1994;331:1286.

17. Bassols A, Massague J. Transforming growth factor $b$ regulated the expression and structure of extracellular matrix chondroitin / dermatan sulfate proteiglycans. J. Biol Chem 1988;263:3039.

18. Ignotz RA, Massague J. Transforming growth factor $b$ stimulates the expression of fibronectin and collagen and their incorporation in the extra cellular matrix. J Biol Chem 1986;261:4337.

19. Edwards DR, Murphy G, Reynolds JJ. Transforming growth factor b stimulates the expression of collagenese and metalloproteinase inhibitor. EMBO J 1987;6:1899.

20. Peltonen J, Hsiao LL, Jakkola S. Activation of collagen gene expression in keloid: Co-localization of type I and IV collagen and transforming growth factor b1. J Invest Dermatol 1991;97:240.

21. Anscher MS, Peters WP, Reisenbichler H. Transforming growth factor $b$ as a predictor of liver and lung fibrosis after autologous bone marrow transplantation for advanced breast cancer. N Engl J Med 1993;328:1592.

22. Pannu R, Tredget TE, Iwashina T. The role of systemic interferoneb2b on plasma TGF-b and histamine levels in hypertrophic scar patients following thermal injury. Proc. Am Burn Assoc 1995;27:60.

23. Meenakshi J, Ramakrishnan KM, Jayaraman V, Chandrashekar S, Babu M. Etiology and management of ear lobule keloid in south India. Plast Reconst Sur 2005 (accepted and in press). 